\title{
Development of Infrared Focal Plane Arrays
}

\author{
Masafumi Kimata ${ }^{*}$ \\ College of Science and Engineering, Ritsumeikan University \\ 1-1-1 Noji-higashi, Kusatsu, Shiga 525-8577, Japan \\ (Received November 9, 2017; accepted February 28, 2018)
}

Keywords: infrared imaging, quantum infrared detector, thermal infrared detector, charge transfer device, MEMS

Almost 40 years have passed since I started in infrared focal plane array (IRFPA) research and development at Mitsubishi Electric Corporation in 1980. Although our path was not in the mainstream, we developed original IRFPA technologies and fabricated products with our IRFPAs for both cooled and uncooled devices. In this paper, I introduce our accomplishments, including PtSi Schottky-barrier (SB) IRFPAs and silicon-on-insulator (SOI) diode uncooled IRFPAs.

\section{Introduction}

I have spent almost 40 years in infrared focal plane array (IRFPA) research and development since 1980. During that period, we experienced a great change in the middle of the 1990s. Before that, infrared imaging was dominated by cooled IRFPAs. We developed PtSi Schottkybarrier (SB) IRFPAs ${ }^{(1)}$ as a cooled IRFPA in that period although it was not a common approach, and commercialized staring IR cameras for civilian applications.

Before 1992, uncooled IRFPAs failed to attract much attention. They leaped into the spotlight when Wood et al. reported the amazing result of a MEMS-based uncooled IRFPA, ${ }^{(2)}$ that had sufficient performance for almost all civilian applications. The impact of this success was too great for the cooled IRFPA to retain its position, and the uncooled IRFPA has since occupied the leading role in the civilian infrared imaging business. Recognizing that situation, we shifted to uncooled IRFPAs. Instead of choosing the dominant microbolometer ${ }^{(2)}$ technology, we devised and developed the minor but more original silicon-on-insulator (SOI) diode technology.

In this paper, I introduce technologies for both cooled and uncooled IRFPAs, that were developed at Mitsubishi Electric Corporation. The topics include the development and commercialization of PtSi SB IRFPAs and the creation and progress of the SOI diode uncooled IRFPA.

*Corresponding author: e-mail: kimata@se.ritsumei.ac.jp http://dx.doi.org/10.18494/SAM.2018.1802

This article is a modified version of the article "My life in IRFPA R\&D" written by Masafumi Kimata published in Proceedings of SPIE Vol. 10177 (Infrared Technology and Applications XLIII) (2017). 


\section{Cooled IRFPAs}

\subsection{Background}

The popular approach to the fabrication of cooled (quantum) IRFPAs has been to use narrowgap semiconductors, such as mercury cadmium telluride. Photodetectors made of narrow-gap semiconductors operate in the intrinsic mode and offer high quantum efficiency, but it had been difficult to increase the spatial resolution of IRFPAs until recently, because of their material characteristics and hybrid focal plane array (FPA) structure.

$\mathrm{Si}$ is a material suitable for large-scale integration. However, Si has a bandgap energy of $1.1 \mathrm{eV}$, and thus if we operate $\mathrm{Si}$ detectors in the intrinsic mode, their cutoff wavelength cannot be extended beyond $1.1 \mu \mathrm{m}$. An SB detector on Si operating in an internal photoemission mode has a longer cutoff wavelength compatible with thermal imaging if we choose proper combinations of materials. For example, PtSi SB on p-type Si has a barrier height of $0.2 \mathrm{eV}$, which corresponds to a cutoff wavelength of $6 \mu \mathrm{m}$, and it can be used as a middle-wavelength infrared (MWIR) detector.

Although the concept of internal photoemission was used for the measurement of SB heights and the evaluation of the energy losses of excited carriers in metals in the $1950 \mathrm{~s},{ }^{(3)}$ serious efforts to use internal photoemissions for infrared detection began in the late 1960s. An example is an experiment conducted by Shepherd and Yang at the US Rome Air Development Center (RADC) in 1971. ${ }^{(4)}$ They fabricated a metal silicide retina on a silicon wafer for an infrared vidicon, and found that metal silicide retinas had extremely high uniformity in responsivity. Since high uniformity is indispensable for high-background terrestrial infrared imaging, and Si-based technology enables large-scale integration with lower cost, they proposed a silicon-based monolithic SB IRFPA in $1973 .^{(1,5)}$ During the 1970 s, however, we saw some limited research activities on PtSi SB infrared charge-coupled devices (IRCCDs) by the Kosonocky group at the RCA David Sarnoff Research Center in collaboration with RADC. ${ }^{(6)}$

\subsection{Beginning}

We started research on IRFPAs at Mitsubishi Electric Corporation in 1980. Considering our company's research facilities and proprietary technologies, we chose IRFPA technology with a PtSi SB detector, which was not a common approach.

The first IRFPA we developed was a $32 \times 64$ PtSi SB IRFPA. On the basis of a US air force report, ${ }^{(7)}$ we designed and fabricated a device, the pixels of which are shown in Fig. 1, and within one year, we obtained the image shown in Fig. $2 .{ }^{(8)}$ Even though it is a low-resolution dotted image, this result was sufficiently promising. It is an uncorrected image with which we confirmed the high uniformity of the PtSi SB detector.

\subsection{Toward higher spatial resolution}

One of the most important advantages of PtSi SB IRFPAs is their monolithic structure that can be fabricated using standard Si LSI process technology. We used the same research and 


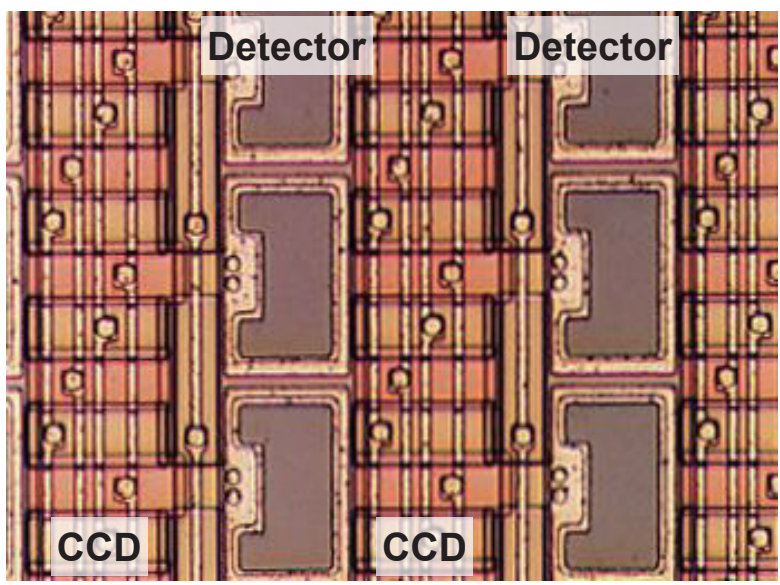

Fig. 1. (Color online) Pixel photo of $32 \times 64 \mathrm{PtSi} \mathrm{SB}$ IRFPA.



Fig. 2. First thermal image taken with $32 \times 64 \mathrm{PtSi}$ SB IRFPA.

development facilities as for dynamic random-access memories, which fueled Si LSI technology in the 1980s. After confirming the potential of PtSi SB technology with the $32 \times 64$ IRFPA, we concentrated on enlarging the array format by exploiting Si LSI process technology and developed a $256 \times 256$ IRFPA in $1983,{ }^{(9)} 512 \times 512$ IRFPA in $1987,{ }^{(10,11)}$ and $1040 \times 1040$ IRFPA in 1991. ${ }^{(12)}$ The last device was the first megapixel FPA for thermal imaging. These devices are shown in Fig. 3 and the trend of increasing spatial resolution (pixel numbers) is shown in Fig. 4, compared with other technologies. For about ten consecutive years from 1983, PtSi SB IRFPA technology surpassed its competition.

\subsection{Charge sweep device}

In increasing the spatial resolution, we reduced the pixel pitch from $133 \times 80 \mu \mathrm{m}^{2}$ for $32 \times$ 64 IRFPA to $37 \times 31 \mu \mathrm{m}^{2}$ for $256 \times 256$ IRFPA, $26 \times 20 \mu \mathrm{m}^{2}$ for $512 \times 512$ IRFPA, and $17 \times$ $17 \mu \mathrm{m}^{2}$ for $1040 \times 1040$ IRFPA. If conventional CCD readout technology is employed for such small pixel pitches, a monolithic structure gives rise to two problems: a lower fill factor and a lower charge-handling capacity. Since we considered conquering these problems to be the most important issue to address, we devised a charge transfer system named a charge sweep device (CSD). ${ }^{(10,11)}$

Figures 5 and 6 show the construction of a CSD FPA and charge transfer in the CSD, respectively. The CSD is a line-addressed interline CCD image sensor in which the vertical charge transfer device operates like a drain with a large charge-handling capacity, even if its width is minimized. After 1987, the CSD readout architecture was applied to the PtSi SB IRFPAs developed at Mitsubishi Electric Corporation. After successful development of our $512 \times 512$ IRFPA, several companies entered the field of PtSi SB IRFPAs. Table 1 summarizes the high-resolution PtSi SB IRFPAs that were developed after 1987. Note that IRFPAs with the CSD readout architecture have larger fill factors and larger maximum charges. 


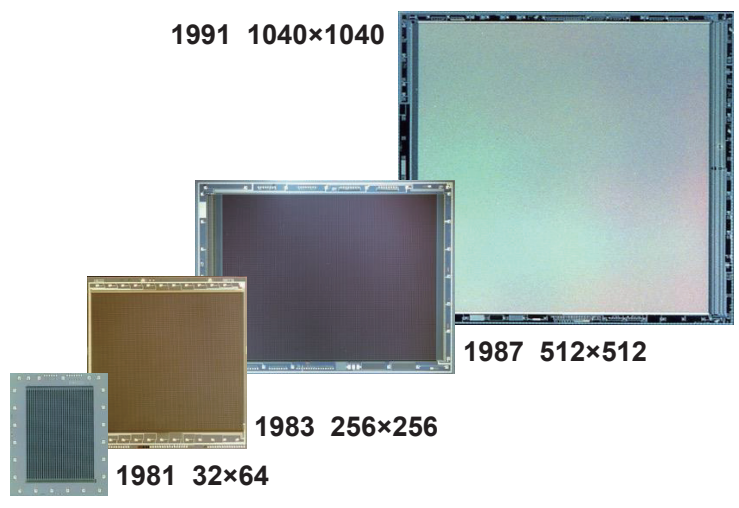

Fig. 3. (Color online) PtSi SB IRFPAs developed at Mitsubishi Electric Corporation.

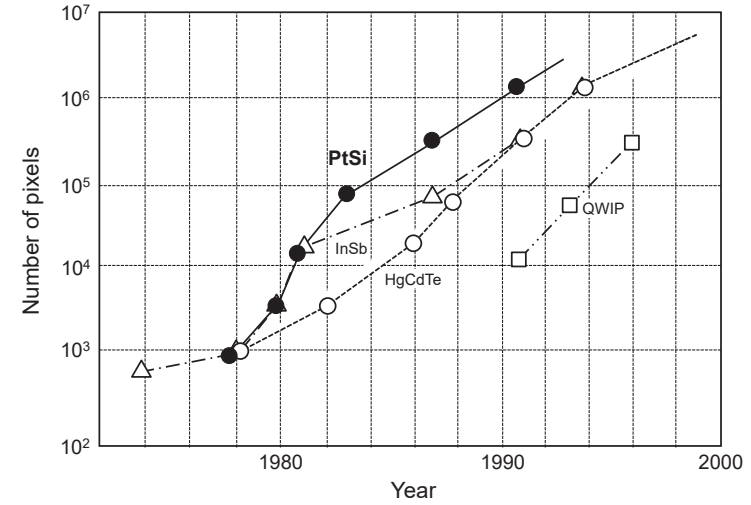

Fig. 4. Trend in spatial resolution of PtSi SB IRFPAs, compared with other IRFPAs.

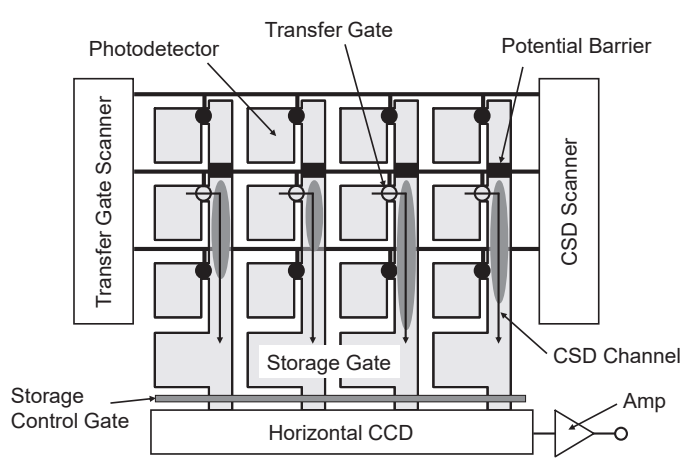

Fig. 5. Construction of FPA with charge sweep device.

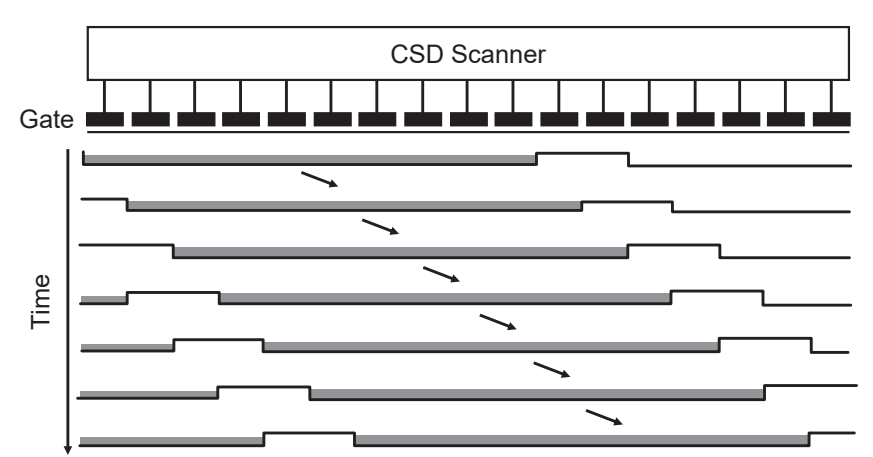

Fig. 6. Charge transfer in charge sweep device, showing time sequential change in channel potential.

Table 1

High-resolution PtSi SB IRFPAs.

\begin{tabular}{lcccccccc}
\hline Array Format & Readout & $\begin{array}{c}\text { Pixel Pitch } \\
\left(\mu \mathrm{m}^{2}\right)\end{array}$ & $\begin{array}{c}\text { Fill Factor } \\
(\%)\end{array}$ & $\begin{array}{c}\text { Maximum } \\
\text { Charge }\end{array}$ & NETD (f/\#) & Year & Company & Reference \\
\hline $512 \times 512$ & CSD & $26 \times 20$ & 39 & $1.30 \mathrm{Me}$ & $0.07 \mathrm{~K}(1.2)$ & 1987 & Mitsubishi & 10,11 \\
$512 \times 488$ & ILCCD & $31.5 \times 25$ & 36 & $0.55 \mathrm{Me}$ & $0.07 \mathrm{~K}(1.8)$ & 1989 & Loral Fairchild & 13 \\
$640 \times 486$ & LACA & $30 \times 30$ & 54 & $0.40 \mathrm{Me}$ & $0.10 \mathrm{~K}(1.8)$ & 1989 & EG\&G Reticon & 14 \\
$512 \times 512$ & ILCCD & $30 \times 30$ & 54 & $0.55 \mathrm{Me}$ & $0.10 \mathrm{~K}(2.8)$ & 1990 & Eastman Kodak & 15 \\
$640 \times 480$ & MOS & $24 \times 24$ & 38 & $1.50 \mathrm{Me}$ & $0.06 \mathrm{~K}(1.0)$ & 1990 & RCA & 16 \\
$640 \times 488$ & ILCCD & $21 \times 21$ & 40 & $0.50 \mathrm{Me}$ & $0.10 \mathrm{~K}(1.0)$ & 1991 & NEC & 17 \\
$640 \times 480$ & HB/MOS & $20 \times 20$ & 80 & $0.75 \mathrm{Me}$ & $0.10 \mathrm{~K}(2.0)$ & 1991 & Hughes & 18 \\
$1040 \times 1040$ & CSD & $17 \times 17$ & 53 & $1.60 \mathrm{Me}$ & $0.10 \mathrm{~K}(1.2)$ & 1991 & Mitsubishi & 12 \\
$512 \times 512$ & CSD & $26 \times 20$ & 71 & $2.90 \mathrm{Me}$ & $0.03 \mathrm{~K}(1.2)$ & 1992 & Mitsubishi & 19 \\
$656 \times 492$ & ILCCD & $26.5 \times 26.5$ & 46 & $0.80 \mathrm{Me}$ & $0.06 \mathrm{~K}(1.8)$ & 1993 & Loral Fairchild & 20 \\
$640 \times 480$ & HB/MOS & $24 \times 24$ & 60 & $1.2 \mathrm{Me}$ & $0.10 \mathrm{~K}(1.4)$ & 1996 & AEG & 21 \\
$811 \times 508$ & ILCCD & $18 \times 21$ & 38 & $0.75 \mathrm{Me}$ & $0.06 \mathrm{~K}(1.2)$ & 1996 & Nikon & 22 \\
$801 \times 512$ & CSD & $17 \times 20$ & 61 & $2.10 \mathrm{Me}$ & $0.04 \mathrm{~K}(1.2)$ & 1997 & Mitsubishi & 23 \\
\hline
\end{tabular}

Charge Sweep Device (CSD), Interline CCD (ILCCD), Line-Addressed Charge Accumulation (LACA), Metal Oxide Semiconductor (MOS), and Hybrid (HB). 


\subsection{Commercialization and applications}

Since we thought that the spatial resolution and sensitivity of the $512 \times 512 \mathrm{PtSi} \mathrm{SB}$ IRFPA were sufficient for commercial applications, in 1987 we initiated a project for the commercialization of a staring infrared camera using the device. A year later, we demonstrated our first commercial product, called IR-5120A (Fig. 7), at an exhibition. This infrared camera became the world's first commercial infrared staring camera.

From 1988 to 2002, we brought seven models to the market, including camera cores for gimbal-mounted systems. All the models used Stirling cycle cryocoolers to cool IRFPAs to a temperature of around $80 \mathrm{~K}$. Although we established the production technology for megapixel IRFPAs, the largest array format of our commercial products was the $801 \times 512$ IR camera IR$\mathrm{M} 700^{(23)}$ because no displays that are compatible with megapixel IRFPAs were readily available in that period. Figure 8 shows an example of an image taken with an IR-M700.

In addition to commercial products, we developed various IR systems for Japanese government and research institutes, and some continue to be used today, testifying to the usefulness of infrared imaging. For example, the Japan Ministry of Defense uses PtSi SB IR cameras on fixed-wing rescue aircrafts, ${ }^{(24)}$ and the Japanese Coast Guard also deploys many patrol vessels equipped with PtSi infrared camera systems.

Since PtSi SB detectors have sensitivity in the short-wavelength infrared (SWIR) spectral region, earth observation is another critical application for PtSi SB IRFPAs. The Japan Earth Resources Satellite-1 (JERS-1) ${ }^{(25)}$ and the Earth Observing Satellite-AM1 (EOS-AM1) ${ }^{(26)}$ used our PtSi SB long linear arrays ${ }^{(27,28)}$ for their SWIR radiometers. Since astronomical observation requires large-format IRFPAs, some Japanese astronomers paid great attention to PtSi SB technology. An example of astronomical application is the SWIR camera of the Venus Climate Orbiter AKATSUKI (Planet-C), ${ }^{(29)}$ which used our 1040×1040 PtSi SB IRFPA.



Fig. 7. (Color online) First commercial staring infrared camera IR-5120A with $512 \times 512 \mathrm{PtSi}$ SB IRFPA.

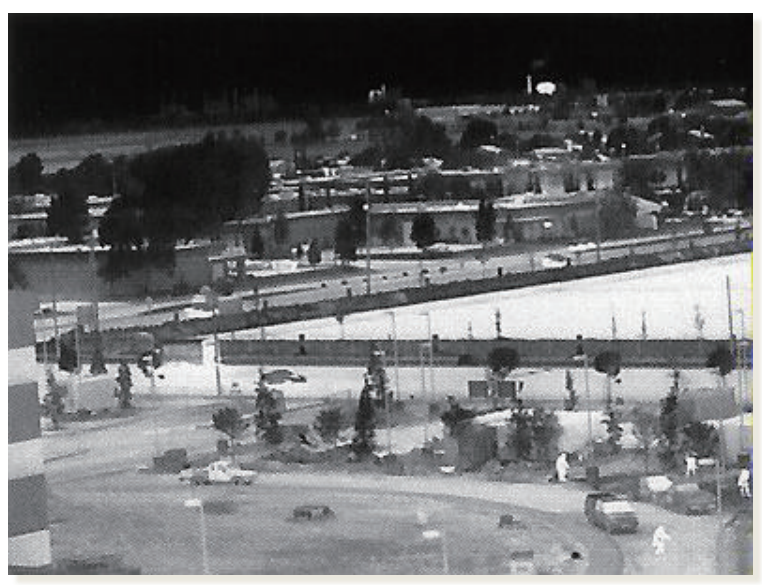

Fig. 8. Example of thermal image taken with IR-M700 using $801 \times 512$ PtSi SB IRFPA. 


\section{Uncooled IRFPAs}

\subsection{Background}

Uncooled IRFPAs use thermal infrared detectors that convert infrared energy to thermal energy, and finally, they obtain electric signals from thermometers as temperature changes. In the 1970s and 1980s, uncooled IRFPAs were constructed with hybrid structures of a pyroelectric detector chip and silicon readout IC, and thus the sensitivity was limited by thermal conduction through the metal bumps of the hybrid structure. Honeywell developed a new technology based on MEMS technology ${ }^{(2)}$ and greatly improved the sensitivity by reducing the thermal conductance of the pixel structure. The technology seemed to satisfy the needs of most commercial applications. Because of the emergence of such high-performance uncooled IRFPAs, we had to abandon PtSi SB IRFPA technology and moved on to the uncooled IRFPA field. We initiated an uncooled IRFPA project in 1993.

The technology developed by Honeywell was a resistance bolometer IRFPA. Although most of the players who entered the uncooled field after Honeywell's success adopted the resistance bolometer (microbolometer) technology, ${ }^{(2)}$ some pursued their own original MEMS-based uncooled IRFPAs, such as thin-film ferroelectric, ${ }^{(30)}$ bimaterial, ${ }^{(31)}$ and thermo-optical types. ${ }^{(32)}$

\subsection{SOI diode uncooled IRFPA}

Our approach was also different from the mainstream. We believed that the microbolometer is not the only technology that could achieve high performance and low cost. In considering alternative devices, we took the strength of our Si LSI technology into consideration and devised structures that could basically be manufactured with our technological assets. After serious discussions, in 1997 we achieved the pixel structure shown in Fig. 9 and named it the SOI diode pixel. ${ }^{(33)}$

In SOI diode pixels, the temperature on the freestanding structure is measured with forwardbiased single-crystal Si $\mathrm{p}-\mathrm{n}$ junction diodes that are thermally isolated and held over a cavity by two thin support legs. The thermal conductance of the support legs is designed to be as small

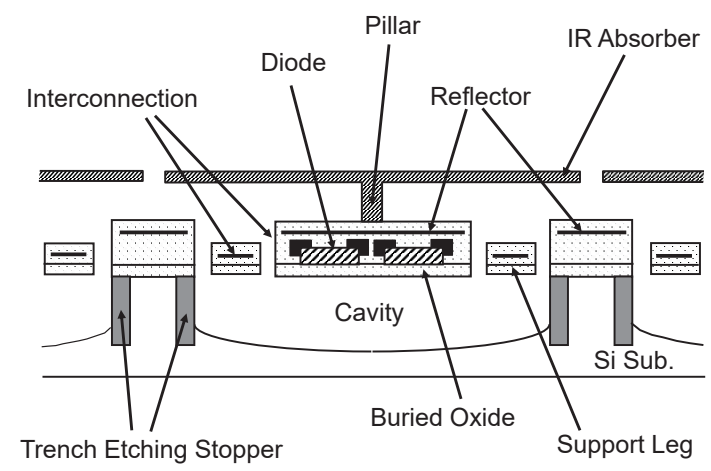

Fig. 9. Cross-sectional structure of SOI diode pixel. 
as possible. Since the diode temperature sensor, the support legs, and the other components in the pixel must be placed on the same level, only a small fraction of the area can be allocated to the freestanding effective infrared-absorbing area if a single-level structure is employed. To overcome this problem, we devised an infrared-absorbing structure that covers almost all of the pixel area. Since the infrared-absorbing structure is thermally connected only to the freestanding diode structure with high thermal conductance, its temperature is essentially identical as that of the diode structure. This infrared-absorbing structure is an interference infrared absorber. Fill factors that approach $90 \%$ are feasible with it. Figure 10 shows an SEM micrograph of the 40- $\mu \mathrm{m}$-pitch pixels of the first-generation SOI diode uncooled IRFPA with $320 \times 240$ pixels. In 1999, we confirmed that the SOI diode uncooled IRFPA have high sensitivities comparable to microbolometers. ${ }^{(33)}$

Figure 11 illustrates a MEMS process for the SOI diode pixel. After the completion of the process for CMOS circuits and temperature-sensing diodes, an organic sacrificial layer

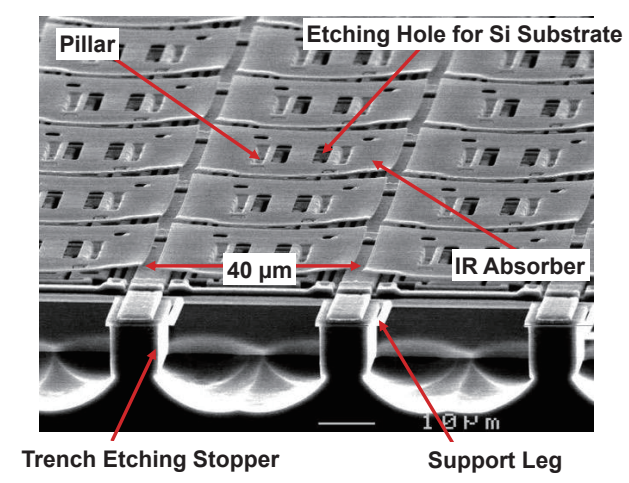

Fig. 10. (Color online) SEM micrograph of 40- $\mu \mathrm{m}$-pitch SOI diode pixel, showing cross section and bird's-eye view.

(a) Deposition of organic sacrificial layer

Sacrificial Layer

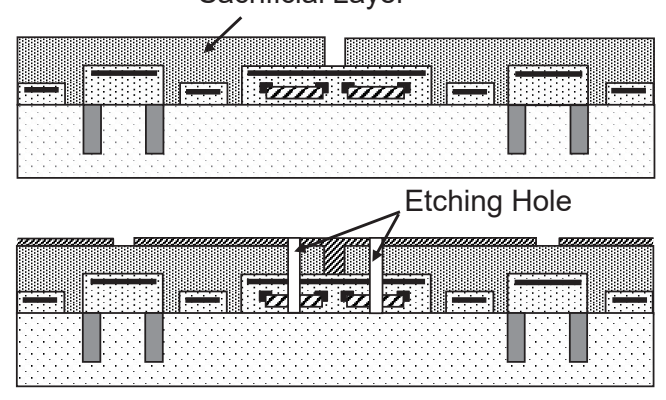

(b) Formation of IR absorber and etching hole

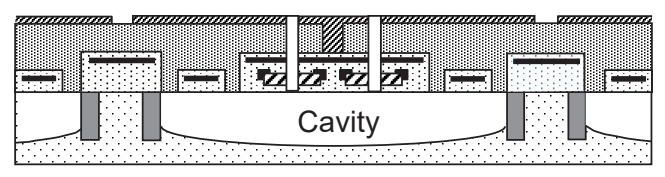

c) Bulk Si etching (Bulk cavity formation)

(d) Release

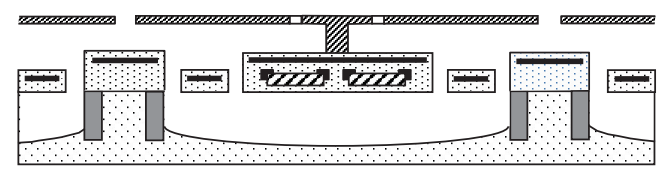

Fig. 11. MEMS process for SOI diode uncooled IRFPA. 
is spin-coated and hardened by baking. A very flat surface is obtained by spin-coating if we choose proper materials and suitable process conditions. Figure 11(a) shows the structure just after patterning of the organic sacrificial layer. Then, the infrared absorber is formed on it. Following infrared-absorber formation, etching holes for bulk silicon etching are opened through the infrared absorber and sacrificial layer [Fig. 11(b)]. After that, bulk silicon etching by $\mathrm{XeF}_{2}$ is performed [Fig. 11(c)]. $\mathrm{XeF}_{2}$ etching is a dry etching process that proceeds isotropically. Therefore, a deep trench-etching stopper surrounding the cavity is needed to avoid connection between neighboring cavities. The buried oxide of the SOI substrate plays the role of the etching stopper to protect the SOI diodes during the $\mathrm{XeF}_{2}$ etching process. Finally, as shown in Fig. 11(d), the organic layers, including the sacrificial layer and the photoresist for etched-hole formation, are removed by the final $\mathrm{O}_{2}$ plasma ashing process.

\subsection{Growth}

As explained above, various alternative technologies have been proposed and developed. Although they challenged the microbolometer position and made considerable inroads, the microbolometer seems unthreatened and continues to hold the leading position in the uncooled IRFPA field. Today, most emerging technologies have already been abandoned.

The SOI diode uncooled IRFPA is a rare exception. Diode temperature sensors have slightly lower sensitivity than resistance bolometers. On the other hand, their noise and uniformity are much better because they are made of single-crystal Si. Furthermore, advanced process technologies for Si LSIs can be utilized when fabricating SOI diode pixels. We believed that the resulting overall performance of SOI diode uncooled IRFPAs could compete with that of microbolometers. In fact, they still survive and have reached a level that is comparable to the microbolometer. Mitsubishi Electric Corporation developed a 25- $\mu$ m-pixel-pitch $640 \times 480$ uncooled IRFPA with a noise equivalent temperature difference (NETD) of $20 \mathrm{mK}$ with $\mathrm{f} / 1.0$ optics in $2008^{(34)}$ and a $15-\mu$ m-pixel-pitch $2 \mathrm{M}$ pixel uncooled IRFPA in $2012^{(35)}$ with SOI diode technology.

Even though SOI diode uncooled IRFPAs are not commercially available at this time, they have been adopted in some government systems. For example, $25-\mu \mathrm{m}$-pixel-pitch $640 \times 480$ SOI diode uncooled IRFPAs are used in the compact infrared cameras (CIRCs) for space applications ${ }^{(36)}$ owing to their high uniformity.

\section{Summary}

Infrared imaging technology has made dramatic progress since we began working in this field around 40 years ago.

The IR-5120A and subsequent series of infrared cameras with PtSi SB IRFPAs stimulated the commercial infrared camera market. This result is significant for the infrared community because the applications of the infrared staring camera were limited to defense and space before its commercialization. From the viewpoint of technology, it is necessary to experimentally show the importance of uniformity in infrared imaging and to demonstrate the usefulness of high-spatial-resolution infrared images. 
The survival of SOI diode technology shows that the microbolometer is not the final technology for uncooled IRFPAs. Of course, microbolometers may continue to occupy the dominant position in the future, but introducing and experimenting with new technologies is also important. Since the available technologies for production often change, another technology might replace the microbolometer. Without any challenges, however, that will never happen.

Our path was never the mainstream; instead we swam in the minor tributaries of both PtSi SB and SOI diode uncooled IRFPAs. Our choices greatly reflected the day's available fabrication technologies, proprietary technologies in our organization, research and development budgets, and social environments. Although I am not positive that our choices were always the best, and admittedly, our technologies only generated tiny waves, we experienced the crest of the progress in the IRFPA field and felt the usefulness of our technologies.

\section{Acknowledgments}

I would like to acknowledge Dr. Natsuro Tsubouchi, Toshiki Seto, and all the others who were involved in the research and development of IRFPAs and infrared imaging systems at Mitsubishi Electric Corporation.

\section{References}

1 F. D. Shepherd and A. C. Yang: Tech. Digest of 1973 IEDM (IEEE, 1973) 310.

2 R. A. Wood, C. J. Han, and P. W. Kruse: Tech. Dig. IEEE Solid-State Sensor and Actuator Workshop (IEEE, 1992) 132.

3 R. Williams: Semiconductors and Semimetals Vol. 6 (Academic Press, New York, 1970) Chap. 2.

4 Private communication with Freeman Shepherd (Feb. 15, 1985).

5 S. A. Roosild, F. D. Shepherd, A. C. Yang, and W. M. Shedd: U.S. Patent 3,902,066 (1975).

6 B. Capone, L. Skolnik, R. Taylor, F. Shepherd, S. Roosild, J. Klein, W. Ewing, and W. Kosonocky: Proc. SPIE 197 (SPIE, 1979) 134.

7 W. F. Kosonocky, E. S. Kohn, and F. V. Shallcross: Final Report RADC-TR-78-259, US Air Force Rome Air Development Center (1978).

8 M. Kimata, M. Denda, T. Fukumoto, N. Tsubouchi, S. Uematsu, S. Shibata, T. Higuchi, T. Saeki, R. Tsunoda, and T. Kanno: Jpn. J. Appl. Phys. 21 (Suppl. 21-1) (1982) 231.

9 M. Kimata, M. Denda, N. Yutani, N. Tsubouchi, H. Shibata, H. Kurebayashi, R. Tsunoda, and T. Kanno: Tech. Digest 1983 ISSCC (IEEE, 1983) 252.

10 M. Kimata, M. Denda, N. Yutani, S. Iwade, and N. Tsubouchi: Tech. Digest 1987 ISSCC (IEEE, 1987) 110.

11 M. Kimata, M. Denda, N. Yutani, S. Iwade, and N. Tsubouchi: IEEE JSSC SC-22 (1987) 1124.

12 N. Yutani, H. Yagi, M. Kimata, J. Nakanishi, S. Nagayoshi, and N. Tsubouchi: Tech. Digest of 1991 IEDM (IEEE, 1991) 175.

13 H. Elabd, Y. Abedini, W. Shieh, J. Kim, M. Shin, J. Chiu, F. Nicol, W. Petro, J. Lehan, M. Duron, M. Mandersons, S. Otto, C. Diaz, S. Lam, H. Balopole, P. Coyle, P. Cheng, and R. Martin: Proc. SPIE 161 (SPIE, 1989) 79.

14 W.-L. Wang, R. Winzenread, B. Nguyen, and J. J. Murrin: Proc. SPIE 1161 (SPIE, 1989) 79.

15 E. T. Nelson, K. Y. Wong, S. Yoshizumi, D. Rockafellow, W. DesJardin, M. Elzinga, J. P. Lavine, T. J. Tredwell, R. P. Khosla, P. Sorle, B. Howe, S. Brickman, and S. Refermat: Proc. SPIE 1308 (SPIE, 1990) 36.

16 D. J. Sauer, F. L. Hsueh, F. V. Shallcross, G. M. Meray, and T. S. Villani: Proc. SPIE 1308 (SPIE, 1990) 81.

17 K. Konuma, S. Tohyama, A. Tanabe, K. Masubuchi, N. Teranishi, T. Saito, and T. Muramatsu: Tech. Digest 1991 ISSCC (1991) 216.

18 J. L. Gates, W. G. Connelly, T. D. Franklin, R. E. Mills, F. W. Proce, and T. Y. Wittwer: Proc. SPIE 1540 (SPIE, 1991) 262.

19 H. Yagi, N. Yutani, S. Nagayoshi, J. Nakanishi, M. Kimata, and N. Tsubouchi: Proc. SPIE 1685 (SPIE, 1992) 37. 
20 D. Wen, J. S. Kim, Y. S. Abedini, and S. S. Yeung: Proc. SPIE 2020 (SPIE, 1993) 36.

21 W. Cabanski, R. Koch, H. Maier, G. Pahler, J. Wedler, J. Ziegler, K. Hofmann, K. Eberhardt, P. Deimel, U. Prechtel, and K. Kapser: Proc. SPIE 2746 (SPIE, 1996) 256.

22 M. Shoda, K. Akagawa, and T. Kazama: Proc. SPIE 2744 (SPIE, 1996) 23.

23 H. Yagi, T. Shiraishi, K. Endo, Y. Kosasayama, M. Kimata, and T. Ozeki: Tech. Digest 15th Sensor Symp. (IEEJ, 1997) 155.

24 JASDF Main Equipment of U-125A, http://www.mod.go.jp/asdf/equipment/kyuunanki/U-125A/ (accessed November 1, 2017) (in Japanese).

25 JAXA, Japanese Earth Resources Satellite "FUYO-1" (JERS-1), http://global.jaxa.jp/projects/sat/jers1/index. html (accessed November 1, 2017).

26 IEEE Xplore, Earth Observing System AM1 mission to Earth, http://ieeexplore.ieee.org/document/700989/ (accessed November 1, 2017)

27 M. Denda, M. Kimata, S. Iwade, N. Yutani, T. Kondo, and N. Tsubouchi: IEEE Trans. Electron Devices 38 (1991) 1145.

28 M. Ueno, T. Shiraishi, M. Kawai, Y. Yoneda, M. Kimata, and M. Nunoshita: Proc. SPIE 2553 (SPIE, 1995) 56.

29 JAXA, Venus Climate Orbitor “AKATSUKI” (PLANET-C), http://global.jaxa.jp/projects/sat/planet_c/ (accessed November 1, 2017).

30 C. M. Hanson, H. R. Beratan, and J. F. Belcher: Proc. SPIE 4288 (SPIE, 2001) 298.

31 R. Amantea, C. M. Knoedler, F. P. Pantuso, V. K. Patel, D. J. Sauer, and J. R. Tower: Proc. SPIE 3061 (SPIE, 1997) 210.

32 M. Wu, J. Cook, R. D. Vito, J. Li, E. Ma, R. Murano, N. Nemchuk, M. Tabasky, and M. Wagner: Proc. SPIE 5783 (SPIE, 2005) 496.

33 T. Ishikawa, M. Ueno, Y. Nakaki, K. Endo, Y. Ohta, J. Nakanishi, Y. Kosasayama, H. Yagi, T. Sone, and M. Kimata: Proc. SPIE 4130 (SPIE, 2000) 152.

34 Y. Kosasayama, T. Sugino, Y. Nakaki, M. Ueno, and K. Kama: ITE Technical Report 32 (ITE, 2008) 21 (in Japanese).

35 D. Fujisawa, T. Maegawa, Y. Ohta, Y. Kosasayama, T. Ohnakado, H. Hata, M. Ueno, H. Ohji, R. Sato, H. Katayama, T. Imai, and M. Ueno: Proc. SPIE 8352 (SPIE, 2012) 83531G-1.

36 E. Kato, H. Katayama, M. Naitoh, M. Harada, R. Nakamura, and R. Sato: Proc. SPIE 8353 (SPIE, 2012) $83533 \mathrm{~J}-1$. 